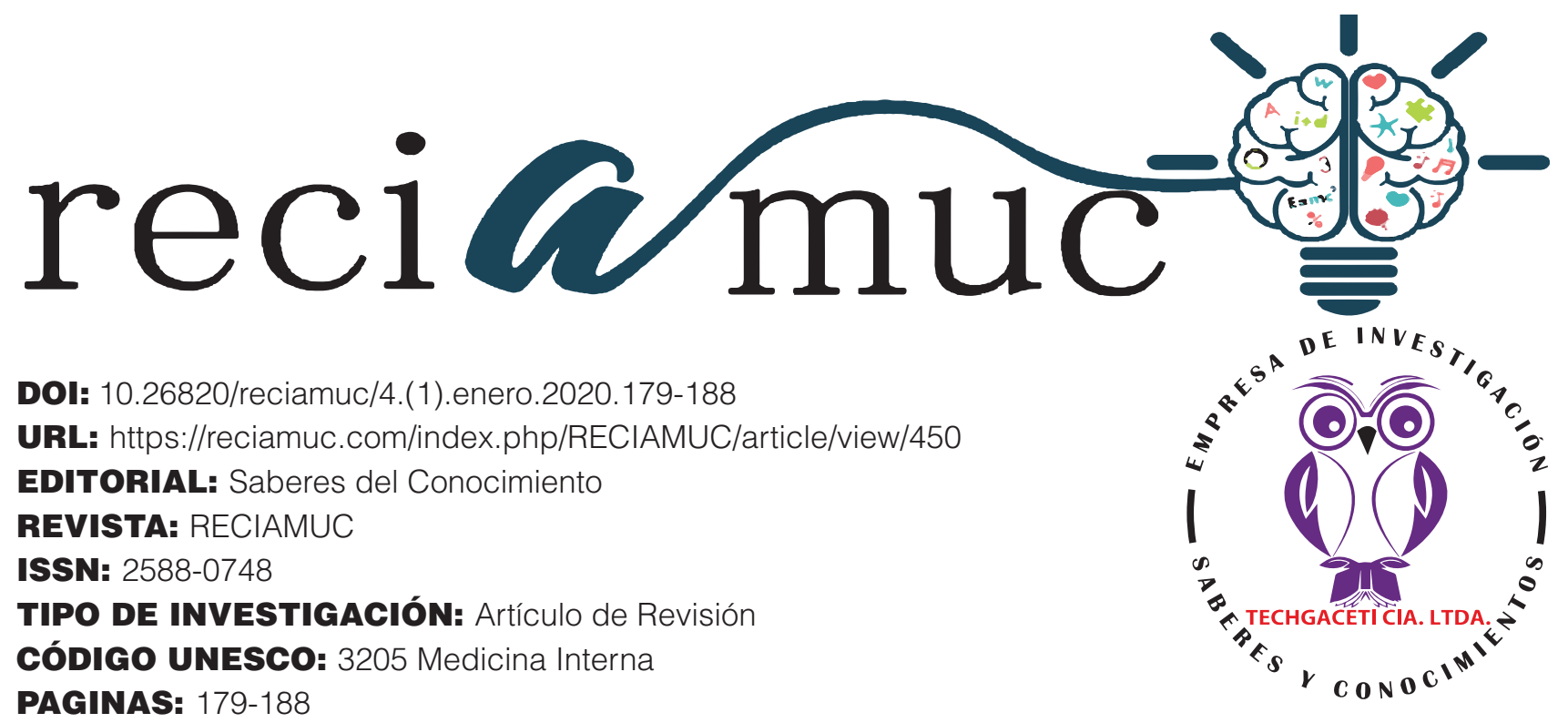

\title{
Antibioticoterapia en pancreatitis aguda
}

Antibiotic therapy in acute pancreatitis

Antibioticoterapia na pancreatite aguda

Luis Javier Alvarez Moyon'; Karol Natalie Galarza Avila2; Karem Lisseth Aguirre Realpe3;

Andrea del Consuelo Torres ${ }^{4}$

RECIBIDO: 18/11/2019 ACEPTADO: 20/12/2019 PUBLICADO: 31/01/2020

1. Médico; Investigador Independiente; Guayaquil, Ecuador; lujam898989@gmail.com; (DD https://orcid.org/00000002-6042-0395

2. Médico; Investigador Independiente; Guayaquil, Ecuador, Ecuador; karito-naty@hotmail.com; (D) https://orcid. org/0000-0003-0458-3584

3. Médico; Investigador Independiente; Guayaquil, Ecuador; Guayaquil, Ecuador; kaguirrerczs5@gmail.com; (D) https://orcid.org/0000-0002-9129-2322

4. Médico; Investigador Independiente; Guayaquil, Ecuador; Guayaquil, Ecuador; andytg_17@hotmail.com; (D) https://orcid.org/0000-0002-6320-0945

\section{CORRESPONDENCIA \\ Luis Javier Alvarez Moyon \\ lujam898989@gmail.com \\ Guayaquil, Ecuador}




\section{RESUMEN}

La pancreatitis aguda es un proceso inflamatorio agudo del páncreas, ocasionado por la activación alterada de las enzimas pancreáticas, con lesión tisular, respuesta inflamatoria local, y compromiso variable de otros tejidos o sistemas orgánicos distantes. Es habitualmente un proceso reversible. La litiasis biliar y el alcohol representan un elevado porcentaje de los casos descritos en las fuentes consultadas. Otras etiologías menos frecuentes son: cirugías, traumatismo, infecciones, hiper trigliceridemia, farmacológicas, hipercalcemia, insuficiencia renal y post colangiopancreatografía retrógrada endoscópica. El diagnóstico de la pancreatitis aguda generalmente se realiza por la presencia de 2 de 3 de los siguientes criterios: dolor abdominal consistente con la enfermedad, amilasa o lipasa sérica elevada más de tres veces del límite superior normal e imágenes fuertemente sugestivas. El dolor usualmente es severo, se presenta en epigastrio o hipocondrio izquierdo con irradiación a espalda o flancos. El uso de la profilaxis antibiótica en el manejo de la pancreatitis aguda, solo ha mostrado beneficio en casos de pancreatitis severa con necrosis del tejido pancreático, definiendo la necrosis como zonas localizadas o difusas de tejido pancreático no viable que, generalmente, se asocian a necrosis grasa peri pancreática. En la tomografía se aprecian zonas con densidad menor que la del tejido normal pero mayor que la densidad líquida y que no incrementan su densidad con el medio de contraste. La administración de antibióticos de amplio espectro con adecuada penetración tisular, es necesaria para prevenir la infección en casos de pancreatitis aguda grave con necrosis pancreática. La metodología usada es descriptiva, con un enfoque documental, es decir, revisar fuentes disponibles en la red, como google académico, con contenido oportuno, actualizado y relevante desde el punto de vista científico que enriquezca el análisis del tema planteado en este artículo.

Palabras clave: Pancreatitis Aguda, Sepsis, Necrosis, Antibiótico, Tomografía Computarizada.

\section{ABSTRACT}

Acute pancreatitis is an acute inflammatory process of the pancreas, caused by altered activation of pancreatic enzymes, with tissue injury, local inflammatory response, and variable involvement of other tissues or distant organic systems. It is usually a reversible process. Biliary lithiasis and alcohol represent a high percentage of the cases described in the sources consulted. Other less frequent etiologies are: surgeries, trauma, infections, hyper triglyceridemia, pharmacological, hypercalcemia, renal insufficiency and post-endoscopic retrograde cholangiopancreatography. The diagnosis of acute pancreatitis is usually made by the presence of 2 of 3 of the following criteria: abdominal pain consistent with the disease, elevated amylase or serum lipase more than three times the normal upper limit and strongly suggestive images. The pain is usually severe, occurs in the left epigastrium or hypochondrium with irradiation to the back or flanks. The use of antibiotic prophylaxis in the management of acute pancreatitis has only shown benefit in cases of severe pancreatitis with pancreatic tissue necrosis, defining necrosis as localized or diffuse areas of non-viable pancreatic tissue that are generally associated with necrosis. peri pancreatic fat. The tomography shows areas with a density less than that of normal tissue but greater than the liquid density and that do not increase its density with the contrast medium. The administration of broad-spectrum antibiotics with adequate tissue penetration is necessary to prevent infection in cases of severe acute pancreatitis with pancreatic necrosis. The methodology used is descriptive, with a documentary approach, that is, reviewing sources available on the web, such as google scholar, with timely, updated and scientifically relevant content that enriches the analysis of the topic raised in this article.

Keywords: Acute Pancreatitis, Sepsis, Necrosis, Antibiotic, Computed Tomography.

\section{RESUMO}

A pancreatite aguda é um processo inflamatório agudo do pâncreas, causado pela ativação alterada das enzimas pancreáticas, com lesão tecidual, resposta inflamatória local e envolvimento variável de outros tecidos ou sistemas orgânicos distantes. Geralmente é um processo reversível. A litíase biliar e o álcool representam uma alta porcentagem dos casos descritos nas fontes consultadas. Outras etiologias menos frequentes são: cirurgias, traumas, infecções, hiper trigliceridemia, farmacológico, hipercalcemia, insuficiência renal e colangiopancreatografia retrógrada pós-endoscópica. O diagnóstico de pancreatite aguda é geralmente realizado pela presença de 2 de 3 dos seguintes critérios: dor abdominal consistente com a doença, amilase elevada ou lipase sérica mais de três vezes o limite superior normal e imagens fortemente sugestivas. A dor é geralmente intensa, ocorre no epigástrio esquerdo ou hipocôndrio com irradiação para as costas ou flancos. O uso da profilaxia com antibióticos no tratamento da pancreatite aguda apenas mostrou benefício nos casos de pancreatite grave com necrose do tecido pancreático, definindo necrose como áreas localizadas ou difusas do tecido pancreático inviável, geralmente associadas à necrose. gordura peri pancreática. A tomografia mostra áreas com densidade menor que a do tecido normal, mas maior que a densidade do líquido e que não aumentam sua densidade com o meio de contraste. A administração de antibióticos de amplo espectro com penetração tecidual adequada é necessária para prevenir a infecção em casos de pancreatite aguda grave com necrose pancreática. A metodologia utilizada é descritiva, com abordagem documental, ou seja, revisando fontes disponíveis na web, como o google scholar, com conteúdo oportuno, atualizado e cientificamente relevante que enriquece a análise do tópico levantado neste artigo.

Palavras-chave: Pancreatite, Sepse, Necrose, Antibiótico, Tomografia Computadorizada. 


\section{Introducción}

La incidencia anual de Pancreatitis Aguda (PA) en diferentes reportes mundiales es de 5 a 80 por 100000 habitantes, aproximadamente variable entre poblaciones. En Sudamérica, particularmente en Brasil se reporta una incidencia de 15.9 casos por 100000 habitantes, en el caso de Perú, los reportes del Ministerio de Salud que incluyen a los trastornos del de páncreas, vesícula biliar y vías biliares indican una incidencia de 28 casos por 100000 habitantes en el 2009 (Acevedo Tizón, Targarona Moden, \& Málaga Rodríguez, 2011).

La pancreatitis aguda se define como una inflamación súbita desarrollada sobre una glándula pancreática inicialmente sana que suele seguirse, si el enfermo sobrevive, de una curación sin secuelas. Esta patología involucra un amplio espectro de enfermedad desde el edema parenquimal leve hasta la pancreatitis severa necrotizante o hemorrágica. La mayoría de los pacientes con pancreatitis aguda desarrollan un curso leve pero cerca de un $15-20 \%$ progresan a pancreatitis aguda severa, definida como la presencia de falla orgánica(shock con presión arterial sistólica menor $90 \mathrm{mmHg}$, hipoxemia con PO2 menor o igual a $60 \mathrm{mmHg}$, insuficiencia renal con creatinina mayor a $2 \mathrm{mg} / \mathrm{dl}$ después de adecuada hidratación, y sangrado gastrointestinal mayor a $500 \mathrm{cc} / 24 \mathrm{hs}$ ) o complicaciones locales como necrosis, absceso y pseudo-quiste la cual está asociada a una tasa de mortalidad del 3-5\% a pesar de los avances en su manejo (Surco, Huerta Mercado, \& Pinto, 2012).

La habilidad para predecir esta severidad puede ayudarnos a identificar pacientes con riesgo incrementado para morbilidad y mortalidad. La severidad del cuadro puede ser señalada usando parámetros clínicos, de laboratorios y radiológicos, indicadores de severidad y marcadores séricos, algunos de estos pueden ser evaluados a la admisión o dentro de las primeras 48 ho- ras, sin embargo el predictor ideal debe ser rápido, reproducible, mínimamente invasivo y exacto especialmente para predecir pacientes con riesgo incrementado de muerte.

Se reconocen dos fases, la fase temprana, que usualmente abarca la primera semana de enfermedad, está marcada por la respuesta sistémica del paciente a la cascada de inflamación, manifestada clínicamente como el síndrome de respuesta inflamatoria sistémica, si la respuesta es persistente, hay riesgo elevado de presentar una falla orgánica en etapa temprana, con alto riesgo de muerte. Sin embargo, la mayoría de pacientes no presenta respuesta sistémica persistente y se recuperan. La fase tardía se caracteriza por la persistencia de signos de inflamación o por la presencia de complicaciones locales, no ocurre en pacientes con pancreatitis leve.

El tratamiento médico de la pancreatitis aguda continúa siendo un manejo de soporte, de tratamiento de las complicaciones y de espera a la resolución del proceso inflamatorio sistémico. Los avances en el manejo médico se basan en el mayor conocimiento de la fisiopatología y el curso clínico como se explicara detalladamente en el siguiente artículo.

\section{Metodología}

Esta investigación está dirigida al estudio "Antibiótico terapia en pancreatitis aguda". Para realizarlo se usó una metodología descriptiva, con un enfoque documental, es decir, revisar fuentes disponibles en la red, como google académico, con contenido oportuno y relevante desde el punto de vista científico para dar respuesta a lo tratado en el presente artículo y que sirvan de inspiración para realizar otros proyectos. Las fuentes consultadas pueden ser estudiadas al final, en la bibliografía.

\section{Resultados}

La pancreatitis aguda es un proceso inflamatorio agudo del páncreas, ocasionado 
por la activación alterada de las enzimas pancreáticas, con lesión tisular, respuesta inflamatoria local, y compromiso variable de otros tejidos o sistemas orgánicos distantes. Es habitualmente un proceso reversible (Nieto-Rodríguez, 2010). Durante un ataque agudo, es probable que las funciones pancreáticas exocrina y endocrina sean anormales. La función endocrina retorna a la normalidad poco después de la fase aguda, mientras que la exocrina, dependiendo de la extensión de la lesión parenquimatosa, puede requerir un año para recuperarse por completo.

Existen argumentos de peso para definir y estratificar la severidad de la pancreatitis aguda. Se comienza por identificar quien requiera tratamiento agresivo agudo, segundo que pacientes necesitan ser trasladados a un centro especializado y tercero quienes los reciben a estos pacientes deben tener un informe o nota, que facilite el clasificarlos como con falla orgánica y/o con complicaciones locales o sistémicas.

Esta nueva clasificación de Atlanta precisa tres grados de severidad (Cerda Cortaza, 2013):

- Pancreatitis aguda leve: se destaca por la ausencia de falla orgánica y de complicaciones locales o sistémicas. Estos pacientes generalmente se egresan durante la fase temprana, no requieren estudios de imagen pancreática y su mortalidad es muy rara.

- Pancreatitis aguda moderadamente severa: se evidencia por la presencia de falla orgánica transitoria o de complicaciones locales o sistémicas en ausencia de falla orgánica persistente.

- Pancreatitis aguda severa: se caracteriza por persistencia de la falla orgánica. Los pacientes con falla orgánica persistente generalmente tienen una o más complicaciones locales. Quien desarroIla falla orgánica persistente en los primeros días tiene un riesgo incrementado de mortalidad de hasta 36-50\% (Cerda Cortaza, 2013). Si a esto se suma necrosis infectada la mortalidad se vuelve extremadamente alta.

- Evolución de la severidad de la pancreatitis aguda: durante la fase temprana, la severidad de la pancreatitis debe reevaluarse diariamente mientras la enfermedad aún está evolucionando. Se recomienda reevaluar entre las 24 - 48 horas y 7 días después del ingreso al hospital.

En la fase tardía de la pancreatitis aguda moderada o severa, las complicaciones locales evolucionan completamente, aunque algunos pacientes con falla orgánica persistente podrían recuperarse sin complicaciones locales. La presencia de infección dentro de las áreas de necrosis es un marcador de incremento de riesgo de muerte. La necrosis infectada sin falla orgánica persistente tiene una menor mortalidad que sí se acompaña de la misma.

\section{Etiología}

La litiasis biliar y el alcohol representan del $60-85 \%$ de los casos (Orellana, 2014). Otras etiologías menos frecuentes son: cirugías, traumatismo, infecciones, hiper trigliceridemia, farmacológicas, hipercalcemia, insuficiencia renal y post colangiopancreatografía retrógrada endoscópica. Es conveniente realizar ultrasonido de abdomen para descartar causa por litos biliares. En caso de que no se encuentren litos y no exista historia de consumo de alcohol, conviene realizar un examen de triglicéridos, sospechando esta etiología si se encuentran en valor mayor a $1000 \mathrm{mg} / \mathrm{dl}$. En pacientes mayores a 40 años se debe considerar un tumor pancreático.

\section{Diagnóstico}

El diagnóstico de la pancreatitis aguda generalmente se realiza por la presencia de 2 de 3 de los siguientes criterios: dolor abdominal consistente con la enfermedad, amilasa o lipasa sérica elevada más de tres 
veces del límite superior normal e imágenes fuertemente sugestivas. El dolor usualmente es severo, se presenta en epigastrio o hipocondrio izquierdo con irradiación a espalda o flancos. Esta descripción es poco específica para la enfermedad, los valores de amilasemia por si solos no son confiables para el diagnóstico, en $20 \%$ de los casos se mantiene dentro de límites normales (Orellana, 2014).

La lipasa pancreática también tiene problemas en cuanto a valor predictivo en ciertas poblaciones, por lo tanto, no es un marcador ideal d esta patología, por ejemplo, en pacientes diabéticos el nivel de lipasa tiende a ser elevado. En general se toma para el diagnóstico una elevación de lipasa o amilasa sérica de 3 - 5 veces el límite superior (Acevedo Tizón, Targarona Moden, \& Málaga Rodríguez, 2011), sin embargo se debe considerar la clínica, y en caso de duda realizar una tomografía axial computarizada con medio de contraste o una colangio resonancia.

Las náuseas y los vómitos están presentes en $80 \%$ de los casos. Puede aparecer febrícula o fiebre. En caso de existir un tercer espacio intra abdominal e importante disminución del volumen, se puede presentar hipotensión y choque (Bisso Andrade, 2011). Ocasionalmente, existe diarrea, hematemesis, confusión, disnea por derrame pleural, atelectasia, insuficiencia cardiaca congestiva o síndrome agudo de dificultad respiratoria.

La tomografía axial computarizada o la resonancia magnética deben ser reservadas para pacientes en los que el diagnóstico es poco claro o que no mejoran su clínica luego de 48-72 horas de la admisión hospitalaria o para evaluar complicaciones (Huerta-Mercado Tenorio, 2013). En todo paciente con cuadro clínico de dolor de gran intensidad en el abdomen, irradiado a la espalda o no, y asociado a náuseas y vómito o no, el clínico debe practicar inmediatamente los exámenes de laboratorio adecuados que, sin tardar y sin dudas, permitan establecer con precisión el diagnóstico exacto. La figura $\mathrm{N}^{\circ} 1$ resume el protocolo de diagnóstico de la pancreatitis aguda.

Figura 1. Algoritmo diagnóstico de la Pancreatitis Aguda

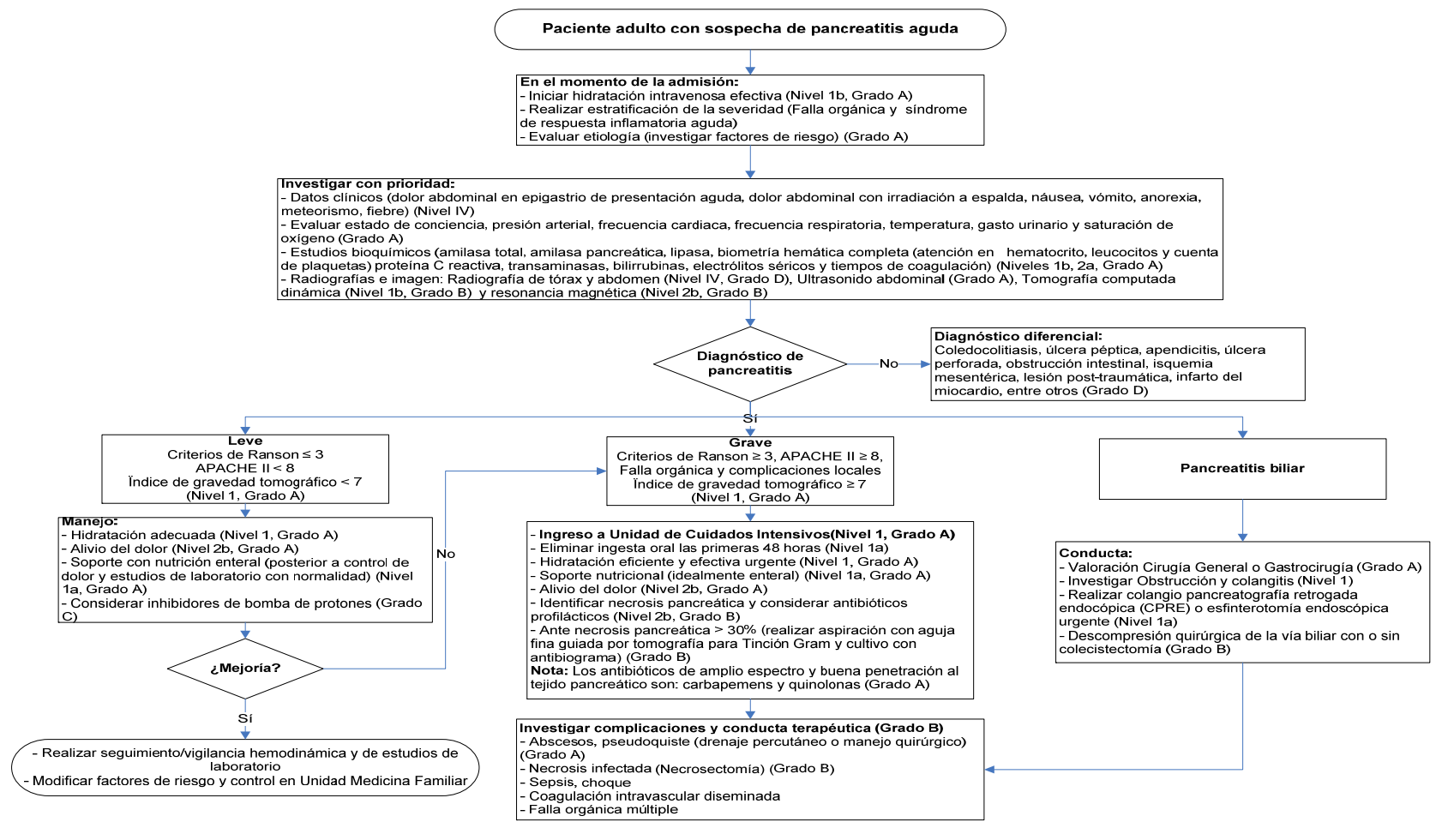

Fuente: (CENETEC, 2009) 
En general, sólo se recomienda seguimiento tomográfico en pacientes con deterioro sistémico, especialmente a aquéllos con puntuación mayor de 4 en el índice de gravedad por tomografía. En quienes se sospecha la presencia de complicaciones asintomáticas, como pseudo quiste o pseudo aneurisma pancreático, se sugie- re obtener la tomografía antes de su salida hospitalaria. Si la suma de la puntuación obtenida en la evaluación de la gravedad según los cambios morfológicos y según el área de necrosis, es mayor de 6 puntos, el paciente debe manejarse en la unidad de cuidados intensivos.

Tabla 1. Índice de gravedad de la pancreatitis aguda por tomografía

\begin{tabular}{|c|c|}
\hline Grado según tomografía & Puntos \\
\hline Grado A: páncreas normal & 0 \\
\hline $\begin{array}{r}\text { Grado B: aumento difuso o focal de la glándula, } \\
\text { atenuación no homogénea. }\end{array}$ & 1 \\
\hline $\begin{array}{c}\text { Grado C: el grado B además de cambios } \\
\text { inflamatorios peri pancreáticos leves. }\end{array}$ & 2 \\
\hline $\begin{array}{r}\text { Grado D: el grado C, más una colección liquida } \\
\text { asociada. }\end{array}$ & 3 \\
\hline $\begin{array}{r}\text { Grado E: dos o más colecciones liquidas o de gas } \\
\text { dentro del páncreas o adyacentes al retro } \\
\text { perineo. }\end{array}$ & 4 \\
\hline
\end{tabular}

Fuente: (Nieto-Rodríguez, 2010)

Complicaciones infecciosas e infección de la necrosis pancreática

Las complicaciones infecciosas extra-pancreáticas como neumonía, infección de catéter venoso central, infección urinaria, entre otras, se manifestaron en el $15 \%$ de los pacientes sin antibiótico profiláctico, mientras que los que recibieron profilaxis la presentaron en el 29\% de los casos según un estudio publicado en Acta de Gastroenterología en Argentina (Barreda, Targarona, \& Milian, 2009). La infección de la necrosis pancreática se comprobó en todos los casos con el cultivo tomado en sala de operaciones de dicho estudio mencionado anteriormente.
Al evaluar la incidencia de infección de la necrosis pancreática se concluyó que no existía diferencia significativa cuando se comparaban los dos grupos, siendo paradójicamente menor en el grupo que no recibió antibiótico-terapia con Imipenem. Los antibióticos deben indicarse cuando hay infecciones extra pancreáticas como neumonías, infecciones de tracto urinario, colangitis, bacteremia, infección de sitio de catéter, y no deben usarse de rutina profilácticamente.

En necrosis infectadas se debe realizar una punción guiada por tomografía para tomar un cultivo, si no existe la opción de punción por aguja fina, mientras se obtiene el resul- 
tado de cultivos, se debe iniciar terapia empírica (carbapenémicos, quinolonas, metronidazol o cefalosporinas a altas dosis). La necrosis infectada se debe sospechar en pacientes con necrosis pancreática o extra pancreática que se deterioran o no mejoran luego de 7-10 días de hospitalización (Mandaria, 2013).

Algunos estudios multi céntricos publicados sobre el uso de antibiótico-terapia profiláctica demuestran que la terapia profiláctica no tendría beneficios en disminuir el índice de infección de la necrosis, tanto pancreática como extra pancreática. Algunos de estos trabajos a pesar de haber sido muy bien diseñados, presentaban algunos problemas en cuanto a la muestra, ya que no todos los pacientes tenían necrosis pancreática o en algunos casos no se aplicaba el antibiótico más idóneo como medida profiláctica o simplemente no estaba estandarizada el tipo de nutrición que recibían estos pacientes, lo cual es muy importante (Nieto-Rodríguez, 2010).

Otros autores critican el alto uso de antibióticos y refieren que el número de pacientes que se necesitaría para un resultado estadístico significativo sería aleatorizar a 1.006 pacientes. Se sugiere utilizar como antibiótico profiláctico el Imipenem para la profilaxis de la necrosis pancreática. Además del antibiótico aplicado a pacientes con necrosis pancreática mayor del 30\% se utilizó Nutrición Enteral Total Temprana que es uno de los factores que podría influenciar más en los resultados, ya que tiene un efecto sobre la translocacción bacteriana en el intestino, siendo éste el principal origen de los microorganismos asociados a la infección de la necrosis pancreática (Bisso Andrade, 2011).

Muchos de los ensayos clínicos de la década pasada, sostenían que la terapia con antibiótico profiláctico era efectiva para evitar la infección de la necrosis pancreática, no podían demostrar que ésta, disminuía el número de pacientes con falla orgánica, el número de pacientes operados ni la mortalidad (Mandaria, 2013). Estos resultados no guardaban relación con la realidad debido a la indicación quirúrgica por la infección de la necrosis, siendo también la principal causa de muerte.

Es importante distinguir que si bien el antibiótico profiláctico no parece efectivo en el tratamiento de la pancreatitis aguda con necrosis, la necesidad de usar antibiótico a libre demanda, es necesaria y efectiva cuando estos pacientes presentan algún tipo de infección. La estancia hospitalaria fue mayor en pacientes que reciben profilaxis.

Esto podría explicar porque la morbilidad y el número de pacientes que requirió tratamiento quirúrgico fueron mayor los resultados en el grupo de pacientes evaluados, según estudio consultado. Es importante recalcar que aquellos médicos que elijan no usar antibiótico profiláctico para los pacientes con necrosis pancreática deben estar prevenidos que podrían necesitarlo como tratamiento en un 33\% de los pacientes, ya sea por infección de la necrosis pancreática o por infección extra-pancreática (Cerda Cortaza, 2013).

Como primera conclusión tenemos que el uso de la profilaxis antibiótica en el manejo de la pancreatitis aguda, solo ha mostrado beneficio en casos de pancreatitis severa con necrosis del 30\% o más del tejido pancreático, definiendo la necrosis de acuerdo a la clasificación de Atlanta como zonas localizadas o difusas de tejido pancreático no viable que, generalmente, se asocian a necrosis grasa peri pancreática (Barreda, Targarona, \& Milian, 2009). En la tomografía se aprecian zonas con densidad menor que la del tejido normal pero mayor que la densidad líquida y que no incrementan su densidad con el medio de contraste. No así en presencia de colecciones liquidas pancreáticas o extra pancreáticas.

Según su penetración a páncreas, los antibióticos se pueden dividir en 3 grupos (Ba- 
llén Parraga, 2010):

Grupo A: Baja penetración. La concentración pancreática no alcanza la concentración mínima inhibitoria de las bacterias presentes. En este grupo se encuentran los aminoglucósidos, la ampicilina y las cefalosporinas de $1^{a}$ generación.

- Grupo B: Penetración moderada. La concentración en páncreas alcanza la concentración mínima inhibitoria de algunas bacterias. En este grupo se encuentran las cefalosporinas de $3^{a}$ generación y las penicilinas de espectro ampliado.

- Grupo C: Alta penetración. Alcanzan concentraciones muy superiores a la concentración mínima inhibitoria de las bacterias sensibles. En este grupo se encuentran las quinolonas, los carbapenemes y el metronidazol.

Dada la complejidad de la pancreatitis aguda, e identificados los dos factores que incrementan la mortalidad, se hace necesario que los pacientes con pancreatitis aguda grave sean atendidos en forma multidisciplinaria en un centro que cuente con Unidad de Cuidados Intensivos, Radiología intervencionista, Endoscopias.

El límite superior recomendado de la profilaxis es 7 a 14 días. La profilaxis contra hongos no se recomienda como rutina, pues los resultados clínicos no demuestran diferencia significativa en cuanto a la reducción de la infección pancreática. Tampoco se recomienda la descontaminación selectiva del tubo digestivo.

No se recomienda profilaxis de rutina en pancreatitis aguda sin confirmación de necrosis, ya que no ha demostrado impacto sobre la morbimortalidad. En pacientes con pancreatitis aguda grave que desarrollan necrosis pancreática, la antibioterapia profiláctica reduce significativamente la sepsis y la mortalidad por todas las causas. El uso de antibiótico terapia profiláctica está reservado para pacientes en quienes se demuestre necrosis superior al $30 \%$ en la tomografía computarizada empleando el esquema definido por la unidad de cuidados intensivos e infectología.

No se ha demostrado que la profilaxis antibiótica de rutina, en pancreatitis aguda sin necrosis, tenga impacto sobre morbimortalidad. La administración de antibióticos de amplio espectro con adecuada penetración tisular, es necesaria para prevenir la infección en casos de pancreatitis aguda grave con necrosis pancreática.

\section{Conclusones}

El dolor abdominal es el síntoma fundamental y está presente desde el comienzo en la mayoría de los ataques de pancreatitis aguda. Aunque puede faltar en bajo porcentaje de pacientes observados, su ausencia puede acompañar a una pancreatitis aguda grave. Puede estar precedido por un cólico biliar o por consumo de alcohol en las 72 horas previas.

En la piel, se puede encontrar ictericia secundaria a coledocolitasis u otra causa de obstrucción de la vía biliar, o compresión de la misma por edema secundario, o incluso por enfermedad hepática coexistente. La prevención y control de la infección y su identificación temprana se convierten en el objetivo principal al evaluar el paciente con pancreatitis aguda.

El diagnóstico clínico se sospecha cuando empeoran los síntomas abdominales y aparece fiebre o aumento de la leucocitosis. Sin embargo, los pacientes con necrosis estéril pueden presentar iguales características, el diagnóstico radiológico es imposible, excepto que se observe gas en el seno de la necrosis, por lo cual se debe realizar con punción-aspiración con aguja fina por ecografía o tomografía computarizada ante la sospecha clínica.

Si el diagnóstico clínico de presunción de pancreatitis aguda no es claro, debe es- 
tablecerse un algoritmo de diagnóstico diferencial clínico; para alguno de los diagnósticos es útil la ecografía, por ejemplo, colecistitis, isquemia intestinal o apendicitis retrocecal. La tomografía computarizada es el método de imágenes más importante para el diagnóstico de la pancreatitis aguda y el estudio de su gravedad. El propósito de administrar el medio intravenoso es distinguir entre la pancreatitis intersticial y la necrosante.

El uso de la profilaxis antibiótica en el manejo de la pancreatitis aguda, solo ha mostrado beneficio en casos de pancreatitis severa con necrosis del tejido pancreático, definiendo la necrosis como zonas localizadas o difusas de tejido pancreático no viable que, generalmente, se asocian a necrosis grasa peri pancreática.

No se recomienda profilaxis de rutina en pancreatitis aguda sin confirmación de necrosis, ya que no ha demostrado impacto sobre la morbimortalidad. En pacientes con pancreatitis aguda grave que desarrollan necrosis pancreática, la antibioterapia profiláctica reduce significativamente la sepsis y la mortalidad por todas las causas. El uso de antibiótico terapia profiláctica está reservado para pacientes en quienes se demuestre necrosis superior al criterio establecido en la tomografía computarizada empleando el esquema definido por la unidad de cuidados intensivos e infectología.

\section{Bibliografía}

Acevedo Tizón, A., Targarona Moden, J., \& Málaga Rodríguez, G. (2011). Identificando a la Pancreatitis Aguda Severa. Revista de Gastroenterología del Perú, 31(3), 236-240. Obtenido de http://www. scielo.org.pe/scielo.php?script=sci_arttext\&pi$d=S 1022-51292011000300006$

Ballén Parraga, H. (2010). Profilaxis antibiótica en pancreatitis aguda: Sí. Revista Colombiana de Gastroenterología, 25(3), 266 - 269. Obtenido de http://www.scielo.org.co/scielo.php?script=sci_arttext\&pid=S0120-99572010000300006

Barreda, L., Targarona, J., \& Milian, W. (2009). ¿Es la antibióticoterapia profiláctica con Imipenem efectiva en los pacientes con necrosis pancreática? Acta de Gastroenterología Latinoamericana, 39(1), 24 - 29. Obtenido de https://www.redalyc. org/pdf/1993/199317369008.pdf

Bisso Andrade, A. (2011). Antibioticoterapia en las infecciones graves. Acta Médica Peruana, 28(1), 27-38. Obtenido de http://www. scielo.org.pe/scielo.php?script=sci_arttext\&pi$d=$ S1728-59172011000100006

CENETEC. (2009). Diagnostico y Tratamiento de la pancreatitis aguda. México: Centro Nacional de Excelencia Tecnológica en Salud.

Cerda Cortaza, L. (2013). Análisis de las nuevas clasifi caciones de la pancreatitis aguda. Cirujano General, 35(1), 16 - 18.

Huerta-Mercado Tenorio, J. (2013). Tratamiento médico de la pancreatitis aguda. Revista Medica Herediana, 24(3), 231-236. Obtenido de http://www. scielo.org. pe/scielo.php?script=sci_arttext\&pi$d=S 1018-130 X 2013000300010$

Mandaria, E. (2013). Últimos avances en pancreatitis agudaLatest advances in acute pancreatitis. Gastroenterología y Hepatología, 36(2), 98 - 102.

Nieto-Rodríguez. (2010). Manejo de la pancreatitis aguda:guía de práctica clínica basada en la mejorinformación disponible. Revista Colombiana de Cirugía, 25(1), 76 - 96.

Orellana, P. (2014). Pancreatitis aguda: revisión de las nuevas guías del 2013. Medicina Legal de Costa Rica, 31(1), 1-5.

Surco, Y., Huerta Mercado, J., \& Pinto, J. (2012). Predicción precoz de severidad en pancreatitis aguda. Revista de Gastroenterología del Perú, 32(3), 241-250. Obtenido de http://www. scielo.org. pe/scielo.php?script=sci_arttext\&pi$d=S 1022-51292012000300002$ 


\section{CITAR ESTE ARTICULO:}

Alvarez Moyon, L., Galarza Avila, K., Aguirre Realpe, K., \& Torres, A. (2020). Antibioticoterapia en pancreatitis aguda. RECIAMUC(4), 179-188. doi:10.26820/ reciamuc/4.(1).enero.2020.179-188

\section{cC) (1) $\$(0)$ BY NC SA}

RECONOCIMIENTO-NOCOMERCIAL-COMPARTIRIGUAL CC BY-NC-SA

ESTA LICENCIA PERMITE A OTROS ENTREMEZCLAR, AJUSTAR Y CONSTRUIR A PARTIR DE SU OBRA CON FINES NO COMERCIALES, SIEMPRE Y CUANDO LE RECONOZCAN LA AUTORÍA Y SUS NUEVAS CREACIONES ESTÉN BAJO UNA LICENCIA CON LOS MISMOS TÉRMINOS. 